\title{
La violence au travail subie par les gestionnaires en sécurité incendie au Québec
}

\author{
Laetitia Larouche $^{a}$, Jacinthe Douesnard ${ }^{b}$
}

RÉSUMÉ. De nombreuses manifestations de violence au travail, tel que le harcèlement, sont aujourd'hui sanctionnées dans plusieurs pays. Considérer cette problématique comme résolue serait toutefois une erreur puisqu'elle affecte encore plusieurs travailleurs, dont certains gestionnaires en sécurité incendie. Devant l'absence de littérature scientifique québécoise portant précisément sur la violence dans ce milieu de travail, il devient nécessaire de décrire les conduites hostiles qui y sont présentes afin de mieux comprendre le phénomène. Cet article expose les résultats d'une étude effectuée selon un devis corrélationnel transversal, dressant un portrait quantitatif de la violence au travail subie par 158 gestionnaires des services incendie du Québec. Les prévalences de violence au travail, les types de comportements hostiles les plus fréquents dans le milieu et certaines caractéristiques des agresseurs seront présentés pour finalement proposer une réflexion sur la violence présente dans les services incendie.

\begin{abstract}
Most manifestations of workplace violence, such as harassment, are now sanctioned in many countries. Considering this issue as resolved would however be a mistake since it still affects several workers, some of whom are managers working in the domain of firefighting. In the absence of scientific literature on workplace violence in this domain in Quebec, it becomes necessary to describe the occurring hostile behaviors to better understand this phenomenon. This article presents the results of a descriptive correlational study providing a quantitative portrait of the workplace violence experienced by 158 managers from the Quebec firefighting domain. The prevalence of workplace violence, the types of hostile behavior most frequently occurring in this domain as well as certain perpetrator's characteristics will be presented to finally suggest a reflection about violence in the fire safety environment.
\end{abstract}

\section{Problématique}

Au Québec, l'importance de la lutte contre les incendies est incontestable. En 2012, 18560 incendies se sont déclarés, blessant pas moins de 217 individus et causant pour 467,9 millions de dollars en dommages. On comptait, à ce moment, 23927 travailleurs œuvrant dans le domaine de la sécurité incendie dont 5529 occupaient les fonctions d'officiers supérieurs (Ministère de la Sécurité publique du Québec, 2013). Les services incendie du Québec sont organisés de manière très structurée et se composent d'une multitude d'échelons. On y retrouve notamment plusieurs chefs (aux opérations, de peloton, de district, etc.), des directeurs, des directeurs adjoints. Chaque travailleur occupe un poste précis aux responsabilités prédéter-

a Doctorante en psychologie organisationnelle, Université de Sherbrooke
b Professeure, Ph. D., psychologue organisationnelle, Université du Québec à Chicoutimi

Revue Organisations \& Territoires $\bullet$ Volume $26 \bullet \mathrm{N}^{\circ} 1-2 \cdot 2017$ minées et reçoit ses directives selon une hiérarchie immuable. Or, cette organisation du travail ne semble pas exempte de conflits. Plusieurs recherches, menées dans différents pays, suggèrent la présence de violence au travail dans ce milieu (Coyne, Craig et Chong, 2004; Rosell, Miller et Barber, 1995; Yoder et Aniakudo, 1996). Il existe toutefois un vide dans la littérature scientifique en regard de la violence vécue par les gestionnaires des services incendie du Québec. Source de problèmes de sommeil, d'absentéisme, de symptômes de stress post-traumatique et de bien d'autres troubles psychologiques (Hirigoyen, 2014; Niedhammer, David et Degioanni, 2006; Niedhammer et al., 2009; Nielsen \& Einarsen, 2012; Pearson et al., 2001), les agressions au travail ont suscité l'intérêt de nombreux chercheurs. Une description détaillée des conduites est désormais nécessaire 
afin de différencier les concepts similaires, démarche essentielle à la progression des connaissances dans ce domaine (Dagenais-Desmarais et Courcy, 2014; Pearson et al., 2001; Yang et al., 2014). En conséquence, cette recherche répondra à la question suivante : quelles formes de violence sont vécues par les cadres du milieu incendie au Québec?

\section{Contexte théorique}

\subsection{Un milieu instigateur de conflits}

Dominant annuellement le palmarès de la confiance de la population envers 30 professions et métiers, les pompiers ne sont pas sans susciter gratitude et admiration (Leger Marketing, 2005). Il est vrai que ces travailleurs évoluent dans des conditions singulières. La responsabilité de vies humaines est un fardeau habituel pour ces intervenants dont la moindre erreur peut devenir fatale. Au péril de leur vie, les pompiers se portent au secours des citoyens en cas d'incendies, d'accidents de la route, de catastrophes naturelles, etc. Le domaine de la sécurité incendie est d'ailleurs identifié par plusieurs auteurs comme empreint de facteurs de risques pouvant engendrer des problèmes de santé psychologique (Armstrong, ShakespeareFinch et Shochet, 2014; Douesnard, 2012; Douesnard et Saint-Arnaud, 2011; Haslam et Mallon, 2003). En revanche, malgré qu’il soit constitutif du même continuum de la carrière en incendie, les postes de cadres comprennent des tâches telles que : l'élaboration des plans d'intervention, le contrôle de leur application, la planification du calendrier des activités de formation, la prévention annuelle et diverses tâches administratives liées aux ressources matérielles et humaines (Gouvernement du Canada, 2013). Parallèlement, bien qu'exerçant davantage des tâches administratives, ces travailleurs supervisent parfois le travail sur les lieux d'intervention. Ils agissent à titre de coordonnateurs, pour certains, mais sont surtout imputables du déroulement des opérations au sens de la loi. Ainsi, ils travaillent de pair avec les pompiers pour éteindre les flammes ou encore sauver des victimes lors de tragédies. Tous ces travailleurs n'en demeurent pas moins témoins de scènes à la fois spectaculaires et potentiellement traumatisantes. D'ailleurs, l'importance du soutien social au maintien en santé psychologique de ces travailleurs ne fait aucun doute (Armstrong et al., 2014; Coyne et al., 2004; Douesnard, 2012; Douesnard et Saint-Arnaud,
2011; Haslam et Mallon, 2003). Par chance, les pompiers à temps plein partagent une proximité hors du commun dans la caserne entre les alarmes, et ils développent des collectifs de travail qui leur permettent de se protéger conjointement des risques inhérents au métier, et de maintenir une bonne santé psychologique (Douesnard, 2012; Douesnard et Saint-Arnaud, 2011). Cependant, bien que cette cohésion soit le pilier du bien-être de ces travailleurs, elle complique considérablement la transition de pompier à cadre intermédiaire (chef pompier), passage tumultueux qui devient d'autant plus bouleversant (Dopson et Neumann, 1998; McConville, 2006). En effet, quelques témoignages des chefs en sécurité incendie indiquent un problème de violence ascendante dans le milieu lors dès leur promotion, c'est-à-dire de violence commise par les subalternes à l'endroit de leur nouveau chef pompier. Ce phénomène ascendant de violence est peu documenté potentiellement en raison des réticences des victimes à le dénoncer (Hirigoyen, 2014). Une explication de cette réticence est fournie par Hirigoyen (2014) qui suggère que les victimes craignent que leurs compétences soient remises en cause. Il devient alors difficile d'avoir une idée claire de l'étendue de cette problématique dans le milieu incendie du Québec.

\subsection{La violence et ses manifestations}

L'Organisation internationale du travail définit la violence au travail comme « toute action, tout incident ou tout comportement qui s'écarte d'une attitude raisonnable par lesquels une personne est attaquée, menacée, lésée ou blessée, dans le cadre ou du fait direct de son travail » (2003, p. 4). Par ailleurs, une multitude d'actes inappropriés au milieu de travail sont recensés. Par exemple, les notions de harcèlement moral, d'intimidation, d'abus émotionnels, de mobbing et d'incivilité au travail (barassment, bullying, emotional abuse, mobbing and incivility), bien qu'analogues et substituées dans certains contextes, impliquent des agissements disparates qui s'inscrivent totalement ou partiellement dans le schème de la violence au travail (DagenaisDesmarais et Courcy, 2014). En effet, ces concepts ont tous en commun d'être une attaque volontaire d'un assaillant envers une ou plusieurs victimes. Toutefois, certains auteurs différencient ces catégories non mutuellement exclusives. Considérant la large variété de la nomenclature utilisée pour dési- 
gner les différents types d'assauts en milieu de travail et le caractère essentiel de la distinction des concepts pour faire progresser les connaissances dans ce domaine (Dagenais-Desmarais et Courcy, 2014; Pearson et al., 2001; Yang et al., 2014), cette recherche s'en tiendra à l'étude du mobbing et de l'incivilité puisque ces derniers semblent présents dans le milieu incendie. Pearson et ses collaborateurs (2001) distinguent l'incivilité des autres concepts par la faible intensité des attaques en cause, dont le caractère est ambigu. Le caractère singulier du mobbing relève quant à lui de la fréquence élevée de l'occurrence des assauts, d'intensité faible à moyenne envers une victime qui se voit placée dans une situation d'impuissance (Leymann, 1996).

\subsection{Une typologie des agressions}

Pour établir le portrait d'une situation donnée, il importe de distinguer les concepts grâce à une typologie des agressions. Pour ce faire, plusieurs auteurs suggèrent des éléments distinctifs, profitables à la discrimination des concepts (Griffin et Lopez, 2005; Hirigoyen, 2014; Pinto, 2014; Yang et al., 2014). Parmi ceux-ci figurent : l'identification des protagonistes, les modalités des gestes posés et les intentions des agresseurs.

L'identification des instigateurs d'agressions au travail est nouvellement considérée comme une étape essentielle à la classification des comportements (Barling et al., 2009; Pinto, 2014; Yang et al., 2014). Cette façon de faire implique qu'il faut situer les protagonistes dans le contexte de l'agression, c'està-dire par rapport aux liens entretenus avec la victime et par rapport à ses responsabilités dans l'organisation. Par ailleurs, lors de la classification, Pinto (2014) ainsi que Yang et ses collaborateurs (2014) soutiennent qu'il est d'égale importance de préciser si les protagonistes sont des individus, des groupes ou des organisations ainsi que leur position hiérarchique au sein de la corporation. En congruence avec ces auteurs, Hirigoyen (2014) distingue le harcèlement transversal, impliquant des rapports de force similaires entre deux collègues hiérarchiquement équivalents, du harcèlement vertical, où les rapports de force sont objectivement inégaux, puisque l'un des protagonistes se trouve en position d'autorité institutionnelle sur le second. Aussi, la direction de l'agression dans le cas de harcèlement vertical revêt une importance pour la classification (Hirigoyen, 2014; Pinto, 2014). Ainsi, on distingue : (1) les phénomènes ascendants, où un ou plusieurs subalternes sont instigateurs d'agressions à l'intention de leur supérieur; (2) les phénomènes descendants, où un ou plusieurs membres de l'organisation commettent des gestes harcelants envers leurs subordonnés et; (3) les phénomènes institutionnels, où les comportements inadaptés sont systémiques puisqu'ils relèvent des pratiques managériales de l'organisation et font partie intégrante de sa culture (Hirigoyen, 2014).

Le détail des comportements conflictuels est également un élément profitable à la classification et l'identification de la situation encourue. La consistance temporelle des agissements, par son impact significatif sur la santé physique et mentale de la victime, revêt une importance particulière (Nielsen et Einarsen, 2012; Nielsen et al., 2009). Celle-ci comprend la fréquence des agressions et leur durée, c'est-à-dire le temps écoulé entre la première et la dernière manifestation agressive (Hirigoyen, 2014).

Source du comportement indu, l'intention à l'origine des manifestations agressives permet une meilleure compréhension de la situation même s'il est illusoire d'avoir une idée parfaitement claire de celle-ci (Griffin et Lopez, 2005). Les gestes posés peuvent être instructifs sur les motivations qui sous-tendent l'agression (Hirigoyen, 2014). À cet effet, à partir de plus de 300 entretiens individuels menés dans les années 1980, Leymann a identifié 45 situations de violence psychologique au travail qu'il a classées en 5 catégories selon l'intention visée par l'agresseur : (1) agissements empêchant la personne de s'exprimer; (2) agissements visant à isoler la personne; (3) agissements visant à discréditer la personne dans le travail; (4) agissements visant à déconsidérer la personne auprès des collègues et; (5) agissements visant à compromettre la santé de la personne (Niedhammer et al., 2007). De plus, la typologie de Feschbach (1964) corrobore l'idée selon laquelle l'intention à la base de l'action influence la situation problématique (Solomon et Feshbach, 1964). Celle-ci distingue deux types d'agression : réactive et instrumentale. Dans le premier cas, les agissements sont perpétrés en réaction à un stimulus externe perçu comme une menace et n'ont pour objectif que l'agression en elle-même. Alors que dans l'autre cas, ces comportements sont un instrument, une méthode en vue d'atteindre un objectif qui dépasse l'action et s'inscrit dans un contexte pour l'agresseur. 


\subsection{Une visée exploratoire}

Considérant la prépondérance de violence au travail dans certains milieux incendie, le peu de littérature sur la violence ascendante soupçonnée dans les services incendie de la province du Québec, et les lourdes conséquences sur la santé psychologique et physique des victimes, la présente recherche a pour objectif de dresser un portrait des situations de violence psychologique à l'égard des chefs de service incendie de la province du Québec.

\section{Méthodologie}

\subsection{Les participants}

Cette recherche ayant pour population les cadres intermédiaires du domaine de l'incendie québécois puisqu'il s'agit des victimes potentielles de la violence ascendante. Les participants à cette recherche ont été rejoints par l'entremise de l'Association des chefs en sécurité incendie du Québec (ACSIQ), qui compte environ 1100 membres dont près de 600 des 732 directeurs des différents services incendie du Québec et 850 chefs actifs. L'échantillonnage de cette étude nous a permis de recruter 158 répondants pour un taux de réponse de 14,4\%. De ces répondants, $71,9 \%$ occupaient ce poste à temps plein ${ }^{1}$ alors que $20 \%$ l'occupaient à temps partiel. Plus de la moitié $(57,5 \%)$ des répondants œuvraient dans des casernes employant majoritairement des pompiers à temps partiel et $26,3 \%$ dans des casernes où œuvrent une majorité de pompiers à temps plein. En tout, 3 femmes, 146 hommes et 9 individus n'ayant pas spécifié leur sexe ont participé à cette recherche. Une majorité de répondants $(95 \%)$ étaient âgés de 35 à 54 ans, possédaient leurs cours d'officier 1 et d'officier $2^{2}(63 \%)$, et plus du tiers d'entre eux avaient suivi une formation universitaire de premier cycle en administration (35\%). Enfin, les répondants étaient répartis selon leurs différents postes conformément aux pourcentages présentés à la figure 1 . On y remarque qu'outre la proportion importante de directeurs ayant répondu au questionnaire, l'échantillon semble représentatif de la population visée par l'étude.

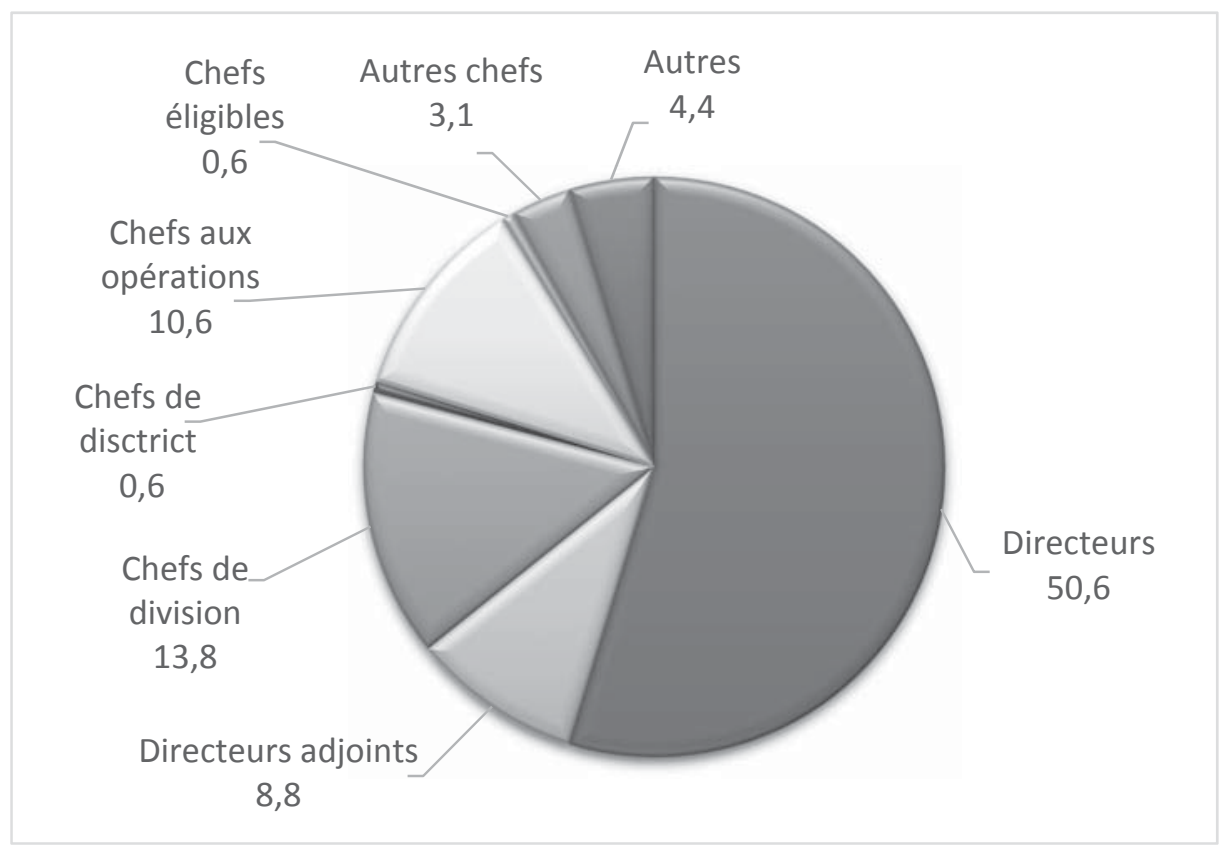

Figure 1 - Répartition de l'échantillon (\%) dans les différents postes de cadres occupés par les répondants 


\subsection{Le questionnaire}

Le questionnaire soumis aux participants comportait trois sections : la première section était composée d'un inventaire de comportements hostiles, soit le Leymann Inventory of psychological terror (LIPT) (Leymann, 1996), la seconde présentait une échelle mesurant la fréquence de comportements d'incivilité auxquels sont exposés les participants : le Workplace Incivility Scale (WIS) (Cortina, Magley, Williams et Langhout, 2001) et la dernière section visait à recueillir des renseignements sociodémographiques.

Le LIPT (Leymann, 1996), traduit de l'anglais au français par Niedhammer et ses collaborateurs (2007), est un inventaire des comportements hostiles déterminant la présence de mobbing au sens de la définition de Leymann (1996). Cet outil permet, dans un premier temps, de recenser la présence de 45 comportements de violence en milieu de travail. Le LIPT classe ces actes inadaptés en cinq catégories mutuellement exclusives : agissements empêchant la personne de s'exprimer (11 items); agissements visant à isoler la personne (5 items); agissements visant à discréditer la personne dans le travail (15 items); agissements visant à déconsidérer la personne auprès des collègues ( 7 items); agissements visant à compromettre la santé de la personne (7 items). Dans un second temps, l'inventaire s'emploie à recueillir des informations au sujet des protagonistes, de la durée et de la fréquence des comportements préalablement recensés et invite les participants à déclarer s'ils ont vécu ou été témoins de violence psychologique au sens de la définition suivante qui leur est proposée :

«Situation où une personne est sujette à des comportements hostiles de la part d'une ou plusieurs personnes de son environnement de travail, qui visent de manière continue et répétée à la blesser, à l'oppresser, à la maltraiter, ou encore à l'exclure ou à l'isoler, pendant une longue période de temps » (Niedhammer et al., 2007, p. 101).

Cet outil permet à la fois de déterminer la quantité d'instigateurs, leur situation hiérarchique, de décrire les comportements de mobbing présents dans le milieu de travail, leur durée, leur fréquence et d'évaluer la prévalence de violence psychologique dans le milieu. De plus, la répartition des comportements selon les cinq catégories permettra de circonscrire les hypothèses concernant les intentions des agresseurs.

Le WIS, développé par Cortina et al (2001)33, est une échelle comportant cinq items correspondant chacun à un comportement d'incivilité. Cet outil mesure la fréquence à laquelle les participants ont dû faire face aux comportements indiqués par ces items (gestes irrespectueux ou condescendants) au cours des cinq années précédant la passation du questionnaire. Les répondants devaient sélectionner la case la plus appropriée selon une échelle de type Likert allant de 1 à $5(1=$ jamais, $2=$ rarement, $3=$ parfois, $4=$ souvent, $5=$ toujours) pour chacun des items. Cet outil est considéré comme ayant une bonne fidélité avec un coefficient alpha de Cronbach de 0,89 (Cortina et al., 2001). Sélectionné notamment pour sa concision, le WIS a pour utilité dans cette recherche de déceler des manifestations de violence en milieu de travail même dans les casernes où le phénomène est présent en intensité moindre. En ce sens, en mesurant la fréquence d'agissements hostiles de faible intensité, il complète le champ d'investigation du LIPT, qui évalue la présence de comportements de moyenne intensité.

Composée de neuf questions et positionnée à la fin du questionnaire, la dernière section comprend les renseignements sociodémographiques réclamés concernant : le sexe, l'âge, le nombre d'années d'expérience dans le domaine, le nombre d'années d'expérience en tant que chef de service incendie, le nombre d'années d'expérience en tant que chef de service incendie au sein de la caserne où le participant travaille actuellement, l'emploi spécifique (chef aux opérations, chefs de peloton ou autres), les modalités de l'emploi (temps plein ou temps partiel), les modalités d'emploi de leurs collègues (temps plein, temps partiel ou mixte) et une question permettant aux participants de nous faire part de leurs commentaires ou précisions.

\subsection{Le déroulement de la collecte}

La recherche a été effectuée selon un devis descriptif corrélationnel transversal sous approbation du comité d'éthique de la recherche de l'Université du Québec à Chicoutimi. Les candidats ont d'abord été rejoints par un courriel envoyé aux membres de l'ACSIQ. Avant de pouvoir accéder au questionnaire informatisé, les participants ont été informés 
des modalités de la recherche. Ceux-ci devaient ensuite donner leur consentement pour accéder au questionnaire informatisé. Une fois remplis, les questionnaires étaient acheminés aux chercheurs pour l'analyse des résultats.

\subsection{L'analyse des données}

Les données recueillies ont été analysées à l'aide du logiciel SPSS 19. Une première étape consistait en une compilation des fréquences pour chacun des renseignements sociodémographiques et un calcul de la répartition, en pourcentage, de l'échantillon selon les catégories de ces mêmes renseignements. Ces résultats ont permis de décrire précisément notre échantillon et d'en évaluer la représentativité par rapport à la population cible, c'est-à-dire le personnel cadre en sécurité incendie du Québec.

En ce qui concerne les informations recueillies à l'aide du LIPT, celles-ci ont été compilées sous forme de fréquence d'apparition pour chaque comportement dont la présence est vérifiée par le questionnaire. Deux méthodes sont suggérées pour obtenir la prévalence de violence psychologique au travail avec le LIPT (Langevin, Boini et Riou, 2012). Il est d'abord possible de considérer que si un participant a dû faire face à au moins l'un de ces comportements, sur une fréquence minimale d'une fois par semaine pendant minimalement six mois, il est effectivement victime de mobbing. Le pourcentage d'individus répondant à ces trois conditions correspond à la première prévalence calculée dans cette étude. Par ailleurs, plusieurs auteurs suggèrent de combiner cette première méthode avec la seconde (Niedhammer, David et Degioanni, 2006; Niedhammer et al., 2009) qui est l'autodéclaration des participants. Ainsi, les participants qui se disent victimes de violence psychologique, à la lecture de la définition qui leur était présentée, ont également été considérés comme des victimes de ce fléau. À des fins de comparaison et pour plus de précision, cette seconde méthode a également été utilisée pour obtenir une prévalence de ce phénomène. Enfin, la prévalence des comportements en fonction de leur fréquence (apparaissant rarement, mensuellement, de tous les jours à une fois par semaine) a été calculée. Ces prévalences seront présentées en comparaison avec celles obtenues lors d'une étude sur la population du sud de la France (Niedhammer et al., 2006) puisque les prévalences obtenues dans cette étude française sont calculées avec le même outil, sur des populations d'hommes comparables. Enfin, une fréquence totale a été calculée pour chaque catégorie de comportements hostiles. Ces calculs ont permis de dresser un portrait quantitatif des comportements présents dans le milieu incendie.

Le résultat du WIS a été calculé par une moyenne des scores par items de même qu'une moyenne générale. Ainsi, la moyenne des scores par items a permis d'identifier les comportements les plus fréquemment vécus alors que la moyenne générale a permis d'estimer la fréquence moyenne à laquelle ces actes surviennent dans le milieu.

\section{Résultats}

\subsection{Comportements de violence dans le milieu incendie}

Le tableau 1 présente les comportements de violence psychologique présents dans le milieu par ordre décroissant de fréquence d'apparition. Ainsi, il est possible de remarquer que les 7 comportements les plus fréquents dans le milieu surviennent à des fréquences supérieures ou égales à $10 \%$. Rappelons que les auteurs du LIPT répartissaient les comportements selon cinq catégories. Ainsi, dans cet échantillon, les gestes les plus fréquemment retrouvés sont ceux inclus dans les catégories « agissements empêchant la personne de s'exprimer » puis dans la catégorie «agissements visant à isoler la personne » alors que les comportements de la catégorie " agissements visant à compromettre la santé de la personne » étaient les moins fréquemment expérimentés. La quantité de comportements retrouvés dans le milieu par catégorie est présentée au tableau 2 . 


\begin{tabular}{l|c}
\multicolumn{1}{c|}{ Comportements de violence } & $\mathbf{( \% )}$ \\
\hline \hline On met vos décisions en question & 21,3 \\
\hline On dit du mal de vous dans votre dos & 18,8 \\
\hline $\begin{array}{l}\text { On ignore votre présence en s'adressant } \\
\text { exclusivement à d'autres }\end{array}$ & 16,3 \\
\hline On propage des rumeurs fausses sur vous & 11,3 \\
\hline Critique permanente à propos de votre travail & 10,6 \\
\hline $\begin{array}{l}\text { Vous êtes constamment interrompu(e) } \\
\text { lorsque vous vous exprimez }\end{array}$ & 10 \\
\hline On fait comme si vous n'existiez pas & 10 \\
\hline Regard ou gestes méprisants & 8,1 \\
\hline $\begin{array}{l}\text { Votre supérieur hiérarchique vous refuse la } \\
\text { possibilité de vous exprimer }\end{array}$ & 7,5 \\
\hline $\begin{array}{l}\text { D'autres personnes vous empêchent de } \\
\text { vous exprimer }\end{array}$ & 7,5 \\
\hline Menaces verbales & 7,5 \\
\hline $\begin{array}{l}\text { On juge votre travail de façon injuste et } \\
\text { blessante }\end{array}$ & 7,5 \\
\hline On ne vous adresse pas la parole & 6,9 \\
\hline $\begin{array}{l}\text { On vous confie des tâches très inférieures à } \\
\text { vos compétences }\end{array}$ & 6,9 \\
\hline $\begin{array}{l}\text { On vous donne sans cesse de nouvelles } \\
\text { tâches }\end{array}$ & 6,3 \\
\hline On ne veut pas ètre abordé par vous & 5,6 \\
\hline $\begin{array}{l}\text { On vous a mis à un poste de travail qui } \\
\text { vous isole des autres }\end{array}$ & 5 \\
\hline On vous ridiculise devant les autres \\
\hline $\begin{array}{l}\text { On crie après vous, on vous injurie à haute } \\
\text { voix }\end{array}$ & 4,4 \\
\hline On s’adresse à vous seulement par écrit & 4,4 \\
\hline $\begin{array}{l}\text { Critique permanente à propos de votre vie } \\
\text { privée }\end{array}$ & 3,8 \\
\hline On vous confie des tâches sans intérêt \\
\hline On vous soupçonne d’être psychiquement \\
\hline Menaces écrites & 2,5 \\
\hline
\end{tabular}

\begin{tabular}{l|c}
\multicolumn{1}{c|}{$\begin{array}{c}\text { Comportements observés } \\
(\mathbf{\%})\end{array}$} & $\mathbf{( \% )}$ \\
\hline \hline On interdit à vos collègues de vous parler & 1,9 \\
\hline $\begin{array}{l}\text { On ne vous confie aucune tâche, vous } \\
\text { n'avez pas de travail }\end{array}$ & 1,9 \\
\hline $\begin{array}{l}\text { On veut vous contraindre à un examen } \\
\text { psychiatrique }\end{array}$ & 1,9 \\
\hline $\begin{array}{l}\text { Quelqu'un occasionne des dégâts à votre } \\
\text { domicile ou sur votre lieu de travail }\end{array}$ & 1,9 \\
\hline On vous confie des tâches humiliantes & 1,3 \\
\hline $\begin{array}{l}\text { On vous confie des tâches très supérieures } \\
\text { à vos compétences }\end{array}$ & 1,3 \\
\hline $\begin{array}{l}\text { Vous êtes obligé(e) de mener des travaux } \\
\text { qui blessent votre conscience }\end{array}$ & 1,3 \\
\hline $\begin{array}{l}\text { On utilise des violences légères contre vous } \\
\text { à titre d'avertissement }\end{array}$ & 1,3 \\
\hline Harcèlement téléphonique & 0,6 \\
\hline $\begin{array}{l}\text { On imite votre allure, votre voix, vos gestes } \\
\text { afin de vous ridiculiser }\end{array}$ & 0,6 \\
\hline $\begin{array}{l}\text { On attaque vos opinions politiques ou reli- } \\
\text { gieuses }\end{array}$ & 0,6 \\
\hline On attaque ou on se moque de vos origines & 0,6 \\
\hline $\begin{array}{l}\text { On vous injurie dans des termes obscènes } \\
\text { ou dégradants }\end{array}$ & 0,6 \\
\hline $\begin{array}{l}\text { On vous oblige à faire des tâches qui nui- } \\
\text { sent à votre santé }\end{array}$ & 0,6 \\
\hline On vous menace de violences corporelles \\
\hline On se moque d'un handicap que vous avez \\
\hline $\begin{array}{l}\text { On vous fait verbalement des allusions ou } \\
\text { propositions sexuelles }\end{array}$ & 0,6 \\
\hline $\begin{array}{l}\text { Malgré votre mauvaise santé, vous êtes } \\
\text { obligé(e) de faire un travail nuisible à votre } \\
\text { santé }\end{array}$ & 0 \\
\hline Vous êtes physiquement maltraité(e) \\
\hline $\begin{array}{l}\text { On en vient à des agressions sexuelles vous } \\
\text { enver }\end{array}$ & 0 \\
\hline
\end{tabular}

Tableau 1-Comportements de violence dans le milieu et fréquences d'apparition associées

\section{Catégories de comportement}

$\mathrm{Nb}$ de fois observée

\begin{tabular}{l|c}
\hline \hline Agissements empêchant la personne de s'exprimer & 125 \\
\hline Agissements visant à isoler la personne & 54 \\
\hline Agissements visant à discréditer la personne dans le travail & 32 \\
\hline Agissements visant à déconsidérer la personne auprès des collègues & 115 \\
\hline Agissements visant à compromettre la santé de la personne & 11
\end{tabular}

Tableau 2-Nombre de fois qu'un comportement de chaque catégorie du LIPT fut observé 


\subsection{Prévalences}

Plusieurs items de notre questionnaire visaient à évaluer la prévalence de certains comportements de violence, qu'ils soient d'ordre physique ou psychologique. Tout d'abord, $38 \%$ des répondants déclarent avoir vécu l'un des comportements mentionnés dans le LIPT au cours de la dernière année, comme présenté au tableau 1. Parmi ceux-ci, 21,3\% estiment être encore aujourd'hui aux prises avec ces comportements hostiles. Par ailleurs, au sens où l'entend Leymann (1996), les participants ayant été exposés à au moins l'un de ces comportements, à une fréquence minimale d'une fois par semaine, pendant minimalement six mois, sont considérés comme victimes de mobbing. Il s'agit de la première méthode utilisée pour calculer la prévalence dans cette étude. Nous retrouvons donc une première prévalence de $10,1 \%$ de violence psychologique dans ce milieu. Une fois que les individus ne considérant pas être victimes de violence psychologique, au sens de la définition qui leur était proposée sont retranchés de ce pourcentage, $7 \%$ des répondants ont été considérés comme victimes de violence psychologique au travail, ce qui représente la prévalence calculée selon la deuxième méthode (Niedhammer et al., 2009).

Par ailleurs, 31,3\% de notre échantillon considéraient avoir été témoins de violence psychologique au sens de la définition proposée. Ces prévalences sont exprimées au tableau 3, en comparaison avec les prévalences d'une étude de Niedhammer et al. (2006), recueillies avec le même outil et un échantillon de population du sud de la France :

\begin{tabular}{l|c|c}
\multicolumn{1}{c|}{ Méthodes d'analyse } & $\mathbf{( \% )}$ & $\begin{array}{c}\text { (\%) } \\
\text { échantillon } \\
\text { de France }\end{array}$ \\
\hline \hline $\begin{array}{l}\text { Ayant vécu au moins un comportement de violence au cours de la } \\
\text { dernière année. }\end{array}$ & 38 & - \\
\hline $\begin{array}{l}\text { Vivant encore un ou plusieurs comportements de violence au jour } \\
\text { de la passation du questionnaire. }\end{array}$ & 21,3 & - \\
\hline $\begin{array}{l}\text { Ayant vécu de la violence au sens de la définition de Leymann } \\
\text { (1996). }\end{array}$ & 10,1 & 10,95 \\
\hline $\begin{array}{l}\text { Considéré comme victime de violence selon les critères de Leymann } \\
\text { et l'autodéclaration. }\end{array}$ & 7 & 8,78 \\
\hline Considérant avoir été témoin de violence au travail. & 31,3 & 23,82 \\
\hline
\end{tabular}

Tableau 3 - Prévalences de violence en milieu de travail chez les cadres des services incendie québécois et comparaison avec les prévalences obtenues pour la population française Source : Niedhammer et al., 2009

\begin{tabular}{l|c|c}
\multicolumn{1}{c|}{ Fréquence de l'occurrence des comportements de violence } & $\mathbf{( \% )}$ & $\begin{array}{c}(\%) \\
\text { échantillon de } \\
\text { France }\end{array}$ \\
\hline \hline Jamais ou non déclaré & 62,8 & \\
\hline Rarement & 16,3 & \\
\hline Au moins une fois par mois & 10,6 & \\
\hline De tous les jours à une fois par semaine & 10,3 & - \\
\hline
\end{tabular}

Tableau 4 - Fréquence à laquelle sont vécus les comportements de violence psychologique par le personnel cadre des services incendie du Québec 


\subsection{Identification des agresseurs}

En ce qui concerne l'identification des agresseurs par rapport aux victimes, de même que leur nombre et leur position dans le contexte de l'agression une fois les données aberrantes retirées de notre échantillon ${ }^{4}$, le nombre d'agresseurs variait entre 0 et $15(\mathrm{M}=3,11$; ET $=2,85)$. Les agresseurs étaient répartis en fonction de la relation professionnelle entretenue avec leur victime et leur sexe.
De fait, $19 \%$ des agressions se déroulaient entre collègues d'un même niveau hiérarchique alors que $81 \%$ de ces agressions se passaient entre deux niveaux hiérarchiques distincts $37,8 \%$ des comportements sont ascendants et $43,2 \%$ sont descendants). Ces résultats sont présentés à la figure 2 .

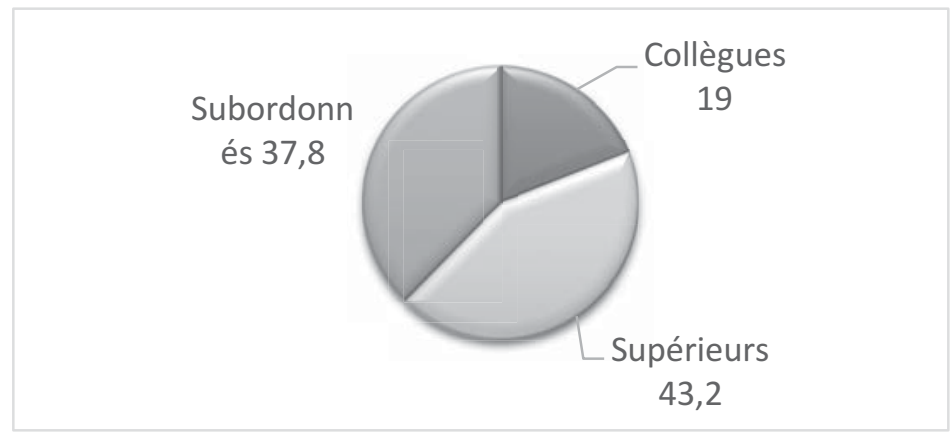

Figure 2 - Répartition des agresseurs (\%) selon leur lien entretenu avec la victime

Par ailleurs, tel qu'exposé à la figure 3, $4 \%$ des agresseurs du milieu sont des femmes, $12 \%$ sont constitués de plus d'un agresseur dont des hommes et des femmes et $84 \%$ sont des hommes.

Groupe composé d'hommes et de femmes

12

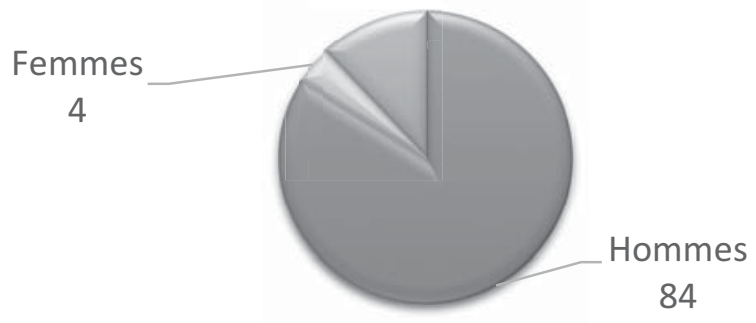

Figure 3 - Répartition (\%) des agresseurs selon leur sexe

\section{$4.4 \quad$ Incivilité}

Le score d'incivilité de l'échantillon est de 1,55 (ET $=0,75)$. Les comportements incivils sont donc, en moyenne, vécus selon des fréquences très faibles, soit entre jamais et rarement sur l'échelle Likert du WIS. Les items les plus fréquemment vécus sont «a prêté peu d'intérêt ou d'attention à votre opinion» et «a douté de votre jugement au sujet d'une tâche dont vous étiez responsable».

\section{Discussion}

\subsection{Comportements de violence dans le milieu incendie}

En se fiant à la catégorisation des comportements de violence de la version française du LIPT, on obtient deux catégories dont les fréquences sont au moins le double de celles obtenues pour chacune des autres catégories. Il s'agit des catégories « agissements empê- 
chant la personne de s'exprimer » et « agissements visant à déconsidérer la personne auprès des collègues ». La combinaison de ces catégories semble congruente avec une hypothèse mentionnée par Hirigoyen (2014), qui stipule que les victimes seraient attaquées sur leurs points névralgiques. En effet, les cadres intermédiaires sont sujets à la solitude puisque soumis sans cesse à des pressions contradictoires entre les différents étages hiérarchiques qu'ils côtoient (Dopson et Neumann, 1998; Harding, Lee et Ford, 2014; McConville, 2006; Sims, 2003). Il s'agit d'un contraste important avec la fraternité régnant au cœur des équipes de pompiers (Armstrong, Shakespeare-Finch et Shochet, 2014; Douesnard, 2012). Il est donc possible de supposer que les cadres intermédiaires du domaine de l'incendie redoutent la solitude. Ainsi, les comportements inclus dans ces catégories peuvent exacerber ce sentiment, si redouté par les chefs. En outre, hypothétiquement, les comportements inclus dans l'une de ces catégories permettent de discréditer l'individu, et ceux inclus dans l'autre catégorie mettent en lumière les comportements qui l'empêchent de se défendre. Toujours en congruence avec cette théorie selon laquelle les victimes sont attaquées sur leurs points névralgiques, les 10 comportements les plus présents dans le domaine inspirent l'exclusion : « on dit du mal de vous dans votre dos »; « on ignore votre présence en s'adressant exclusivement à d'autres »; «on propage des rumeurs fausses sur vous »; « critique permanente à propos de votre travail "; " vous êtes constamment interrompu(e) lorsque vous vous exprimez » et; « on fait comme si vous n'existiez pas ». Chacun de ces comportements a été vécu par $10 \%$ ou plus de l'échantillon au cours de l'année précédant la passation du questionnaire. Dans un corps de métier où le soutien social et la cohésion d'équipe sont si importants (Armstrong et al., 2014; Douesnard, 2012), le fait que les comportements les plus fréquents aient pour objectif d'écarter un individu de son collectif de travail, lequel est essentiel pour le maintien en santé psychologique, est inquiétant.

\subsection{Prévalences}

Les résultats de la présente étude, en matière de prévalence de violence psychologique au travail, sont similaires à ceux de la population du sud de la France avec des écarts de moins de $2 \%$ selon les deux méthodes utilisées pour effectuer le calcul soit : (1) le pourcentage d'individus qui ont vécu au moins un des comportements de violence à une fréquence minimalement hebdomadaire pendant au moins six mois et;
(2) le pourcentage d'individus qui répondent aux critères de la première méthode tout en déclarant avoir vécu de la violence psychologique au sens de la définition qui leur était présentée. Bien que ces résultats soient très similaires à ceux de la France, les résultats obtenus avec ces méthodes indiquent des prévalences légèrement plus élevées en France. D’un autre côté, les prévalences des comportements vécus sur une base hebdomadaire à journalière sont légèrement plus élevées dans l'échantillon de la présente étude que dans l'échantillon français. Les résultats de la population générale de référence utilisée et ceux de cette étude semblent donc équivalents. Toutefois, cette prévalence de violence psychologique dans le domaine de l'incendie demeure élevée. D'ailleurs, elle est plus de 2 fois supérieure à celle obtenue par la population suédoise avec le même outil; $10,1 \%$ chez les chefs du Québec contre 3,5\% pour la population suédoise. Les prévalences de violence psychologique obtenues de $7 \%$ et $10,1 \%$ demeurent donc inquiétantes, surtout en considérant les lourds impacts associés à ce phénomène, conjugués aux responsabilités importantes incombant aux cadres des services incendie québécois (Courcy et Savoie, 2003; Hirigoyen, 2014;Niedhammer, David et Degioanni, 2006; Niedhammer et al., 2009; Nielsen et Einarsen, 2012).

En outre, l'important écart entre le pourcentage d'individus qui se disent témoins de violence psychologique $(31,3 \%)$ et ceux qui s'en disent victimes $(7 \%)$ dans l'échantillon de cette étude porte à réflexion. Cet écart est d'ailleurs bien plus important dans la présente étude (écart de 24,3\%) que l'écart calculé pour la population du sud de la France (écart de 15,04 \%). Quelques hypothèses peuvent être émises pour expliquer cette distinction entre les échantillons. Il est notamment possible de se questionner sur la perception des situations par le personnel cadre des services incendie du Québec. Une situation peut-elle être perçue différemment lorsque les cadres sont impliqués que lorsqu'il s'agit d'un de leurs collègues? Les cadres des services incendie seraient ainsi plus portés à qualifier les situations vécues par les autres de violence psychologique, que celles qu'ils vivent. Par ailleurs, cette différence entre les écarts pourrait également s'expliquer par la grande proximité entre les travailleurs à temps plein du domaine de l'incendie. En effet, il est possible de croire que cette proximité leur permet d'être au fait de la majorité des cas de violence à leur caserne alors que l'information serait moins accessible dans les autres corps de métier. De plus, cela pourrait indiquer 
une culture de violence où les comportements hostiles sont considérés comme normaux, voire positifs comme dans l'étude d'Archer (1999). Ainsi, les répondants admettent être témoins de ces comportements, mais ne les considèrent pas comme violents.

\subsection{Identification des agresseurs}

Le phénomène en présence comprend des situations avec un agresseur simple et d'autres avec plusieurs agresseurs. La répartition des agresseurs selon la relation qu'ils entretiennent avec leur victime permet de déterminer le sens et la direction de celle-ci. En l'occurrence, avec des pourcentages de près de $40 \%$ chacun, ce phénomène peut être qualifié à la fois de violence verticale ascendante et descendante (Hirigoyen, 2014). C'est-à-dire, que les comportements de violence sont à la fois dirigés des subalternes vers leurs cadres intermédiaires et des supérieures hiérarchiques vers leurs subalternes. Les cas de violence psychologique dans le milieu se situeraient donc majoritairement entre deux étages hiérarchiques. Ainsi, il semble peu probable que les comportements de violence soulevés dans notre étude soient partie intégrante du processus de socialisation entre collègues, comme le proposent Coyne et ses collaborateurs (2004), étant donné qu'ils sont effectués par différents niveaux hiérarchiques, contre différents niveaux hiérarchiques. Notons tout de même que le harcèlement horizontal semble également présent puisque $19 \%$ des comportements violents provenaient d'interactions entre collègues.

Enfin, bien que la population et l'échantillon à l'étude soient majoritairement composés d'hommes, les femmes occupent une proportion importante du nombre d'agresseurs. De fait, alors que seulement $2 \%$ de l'échantillon était composé de femmes, $16 \%$ des comportements agressifs étaient commis par des femmes ou groupes incluant des femmes. Ceci n'est pas sans rappeler les résultats de Niedhammer et al. (2006). La prévalence de violence psychologique est pratiquement toujours un peu plus élevée pour les femmes que pour les hommes dans cette étude. Il est toutefois impossible de conclure, avec cette étude, à une différence significative entre les deux populations.

\section{$5.4 \quad$ Incivilité}

En ce qui a trait au score d'incivilité dans l'étude, celui-ci est considéré comme relativement bas avec une moyenne située entre les scores « jamais » et « rarement ». Or, on remarque tout de même une congruence avec les résultats obtenus au LIPT en ce qui concerne les comportements les plus fréquemment vécus dans le milieu. En effet, les items « a prêté peu d'intérêt ou d'attention à votre opinion » et «a douté de votre jugement au sujet d'une tâche dont vous étiez responsable », semblent avoir le même impact que ceux de la catégorie «agissements visant à déconsidérer la personne auprès des collègues » du LIPT.

\subsection{Limites de l'étude}

Cette étude comporte quelques limites, notamment en ce qui a trait à son échantillon. En effet, il contient différents postes et différentes organisations du travail (temps plein, temps partiel, directeur, chefs, etc.) avec des travailleurs qui évoluent dans des conditions disparates et qui, à cause de cette catégorisation, peuvent, à certains égards, être considérés comme des populations distinctes. L'échantillon hétérogène rend difficile les généralisations spécifiques, mais offre un portrait plus représentatif du milieu incendie québécois.

Quelques limites supplémentaires se rapportent à l'outil utilisé. En effet, il est possible que les prévalences soient légèrement surestimées puisque les gestionnaires se sentant interpellés par le sujet pourraient avoir été plus enclins à participer à l'étude. De plus, une des limites de cette étude est inhérente à l'utilisation du LIPT. Cet outil n'étant pas conçu pour calculer des sous-scores aux catégories de violence qu'il recèle, l'utiliser de cette façon ajoute un biais. En effet, puisque les éléments de chaque catégorie sont regroupés dans le questionnaire, il y a un possible effet de halo (Niedhammer et al., 2007), c'est-à-dire que les réponses en chaîne peuvent influencer le répondant et augmenter son score par catégorie. De plus, selon certains auteurs, cet outil est considéré comme plutôt long et répétitif, ce qui peut devenir éprouvant pour le participant et biaiser son résultat (Niedhammer et al., 2007). Enfin, il est raisonnable de penser qu'un biais supplémentaire est présent parce que la population à l'étude est en partie constituée de travailleurs à temps partiel ou en interaction avec des travailleurs à temps partiel. Ainsi, les calculs de fréquence présents dans les deux outils peuvent avoir été évalués à la baisse, tout comme les prévalences de violence psychologique au sens des deux définitions. En effet, on ne peut être victime d'un comportement de 
violence psychologique d'un assaillant tous les jours, si on ne le fréquente pas sur une base journalière ou hebdomadaire.

\section{Conclusion}

En résumé, cette étude a permis, grâce à un questionnaire composé d'un inventaire de comportements de violence psychologique, d'une échelle mesurant la fréquence d'apparition de comportements incivils et de questions sociodémographiques, de dresser un portrait quantitatif des comportements de violence que subit le personnel cadre des services incendie de la province du Québec. Des prévalences de $7 \%$ et 10,1\% de violence psychologique dans le milieu incendie ont été mises en lumière par cette étude. De plus, cette recherche exploratoire suggère que la majorité des comportements présents dans le milieu incendie québécois se rapporte à des actes visant à exclure un individu ou à le discréditer. Ces résultats, dans un milieu où sont prônées des valeurs de coopération, de collaboration et de travail d'équipe, ne sont pas sans susciter de nouveaux questionnements. Par ailleurs, ces données ne permettent qu'une description libre d'interprétations sans offrir la possibilité d'expliquer et de comprendre ces résultats avec certitude. Considérant les impacts importants sur la santé psychologique qu'engendrerait la violence psychologique au travail et considérant les prévalences de ce phénomène dans le milieu incendie, des études exploratoires employant la méthode inductive seraient pertinentes afin de comprendre et de prévenir ces situations.

\section{NOTES}

1 Adaptés à la réalité territoriale du Québec, les services incendie comptent des travailleurs à temps plein et des travailleurs à temps partiel. Les pompiers à temps plein font ce métier comme occupation principale et attendent les interventions en caserne durant leur quart de travail alors que les pompiers à temps partiel ont un emploi principal, qu'ils quittent pour se rendre aux interventions advenant une urgence.

2 Noms donnés aux formations de gestion spécifiques pour les officiers de pompiers.

3 La version du WIS remplie par les participants correspondait à une traduction libre.

4 Les données indiquant que plus de 80 individus étaient impliqués ont été retirées puisqu'il ne s'agit plus d'un phénomène de violence interindividuelle, mais bien d'un phénomène plus important de conflit organisationnel.

\section{RÉFÉRENCES}

Armstrong, D., Shakespeare- Finch, J. et Shochet, I. (2014). Predicting post-traumatic growth and post- traumatic stress in firefighters. Australian Journal of Psychology, 66(1), 38-46.

Cortina, L. M., Magley, V. J., Williams, J. H. et Langhout, R. D. (2001). Incivility in the workplace: incidence and impact. Journal of Occupational Health Psychology, 6(1), 64-80.

Coyne, I., Craig, J. et Chong, P. S. L. (2004). Workplace bullying in a group context. British Journal of Guidance and Counselling, 32(3), 301-317.

Dagenais-Desmarais, V. et Courcy, F. (2014). The deleterious role of workplace violence on personnel engagement, motivation and self-determination The Oxford Handbook of Work. Engagement, Motivation and Self-Determination Theory, 10, 88-100.

Dopson, S. et Neumann, J. E. (1998). Uncertainty, contrariness and the double- bind: middle managers' reactions to changing contracts. British Journal of Management, 9, 53-70.

Douesnard, J. (2012). Métier à risque la santé psychologique des pompiers. Québec, Québec : Presses de l'Université du Québec.

Douesnard, J. et Saint-Arnaud, L. (2011). Le travail des pompiers : un métier au service de l'autre. Travailler, $26(2), 35$.

Gouvernement du Canada. (2013). Chefs et officiers supérieurs des services d'incendie. Service Canada au service des gens.

Griffin, R. et Lopez, Y. (2005). Bad behavior" in organizations: a review and typology for future research. Journal of Management, 31, 988-1005.

Harding, N., Lee, H. et Ford, J. (2014). Who is 'the middle manager'? Human Relations, 67(10), 1213-1237.

Hirigoyen, M.-F. (2014). Le harcèlement moral au travail (1 re éd.). Paris, France : Presses Universitaires de France. 
Keashly, L. et Neuman, J. H. (2008). Final report: workplace behaviour (bullying) project survey. Mankato, MN: Minnesota State University-Mankato.

Langevin, V., François, M., Boini, S.et Riou, A. (2012). Leymann inventory of psychological terror (LIPT). Documents pour le médecin du travail, (129), 101-105.

Leymann, H. (1996). The content and development of mobbing at work. European Journal of Work and Organizational Psychology, $5(2), 165-184$

McConville, T. (2006). Devolved HRM responsibilities, middle-managers and role dissonance. Personnel Review, 35(6), 637-653.

Ministère de la Sécurité publique du Québec. (2013). Rapport d'activité et statistiques sur les incendies déclarés en 2012 La sécurité incendie au Québec, 14-45.

Niedhammer, I., David, S. et Degioanni, S. (2006). Association between workplace bullying and depressive symptoms in the french working population. Journal of Psychosomatic Research, 61(2), 251.

Niedhammer, I., David, S., Degioanni, S., Acquarone, D., Aicardi, F., André-Mazeaud, P., Vital, N. (2007). The french version of the Leymann's questionnaire on workplace bullying: the Leymann inventory of psychological terror (LIPT). Archives des Maladies Professionnelles et de l'Environnement, 68(2), 136-152.

Niedhammer, I., David, S., Degioanni, S., Drummond, A., Philip, P., Acquarone, D., Vital, N. (2009). Workplace bullying and sleep disturbances: findings from a large scale cross-sectional survey in the French working population. Sleep, 32(9), 1211.

Nielsen, M. B. et Einarsen, S. (2012). Outcomes of exposure to workplace bullying: A meta-analytic review. Work \& Stress, 26(4), 309-332.

Nielsen, M. B., Skogstad, A., Matthiesen, S. B., Glasø, L., Aasland, M. S., Notelaers, G. et Einarsen, S. (2009). Prevalence of workplace bullying in Norway: comparisons across time and estimation methods. European Journal of Work and Organizational Psychology, 18(1), 81-101.

Pearson, C. M., Andersson, L. M. et Wegner, J. W. (2001). When workers flout convention: A study of workplace incivility. Human Relations, 54(11), 1387-1419.

Pinto, J. (2014). Expanding the content domain of workplace aggression: A three-level aggressor-target taxonomy. International Journal of Management Reviews, 16(3), 290-313.

Rosell, E., Miller, K. et Barber, K. (1995). Firefighting women and sexual harrassment. Public Personnel Management, 24(3), 339-350.

Sims, D. (2003). Between the millstones: a narrative account of the vulnerability of middle managers' storying. Human Relations, 56(10), 1195-1211.

Yang, L.-Q., Caughlin, D. E., Gazica, M. W., Truxillo, D. M. et Spector, P. E. (2014). Workplace mistreatment climate and potential employee and organizational outcomes: A meta-analytic review from the target's perspective. Journal of Occupational Health Psychology, 19(3), 315-335.

Yoder, J. D. et Aniakudo, P. (1996). When pranks become harassment: The case of African American women firefighters. Sex Roles, 35(5-6), 253-270. 